\title{
What constitutes a high quality discharge summary? A comparison between the views of secondary and primary care doctors
}

\author{
Rowan Yemm¹, Debi Bhattacharya ${ }^{1}$, David Wright ${ }^{1}$, Fiona Poland ${ }^{2}$ \\ ${ }^{1}$ School of Pharmacy, University of East Anglia, UK \\ ${ }^{2}$ School of Allied Health Professions, University of East Anglia, UK \\ Correspondence: Rowan Yemm, School of Pharmacy, University of East Anglia, Norwich Research Park, Norfolk, NR4 7TJ. UK. \\ Email: r.yemm@uea.ac.uk \\ Accepted: June 01, 2014
}

\begin{abstract}
Objective: This study aimed to identify any differences in opinion between UK hospital junior doctors and community General Practitioners (GPs) with respect to the ideal content and characteristics of discharge summaries, and to explore junior doctors' training for and awareness of postdischarge requirements of GPs.
\end{abstract}

Methods: A piloted anonymous survey was posted to 74 junior doctors at a UK general hospital and 153 local GPs. Doctors were asked to rank discharge summary key content and characteristics in order of importance. GP discharge summary preferences and junior doctor training were also investigated. Non-respondents, identified by non-receipt of a separate participation card, were followed up once.

Results: Thirty-six (49\%) junior doctors and 42 (28\%) GPs returned completed questionnaires. Accuracy was a priority with 24 (72\%) GPs and 28 (88\%) junior doctors ranking it most important. Details of medication changes were considered most important by 13 (39\%) GPs and 4 (12\%) junior doctors. Inadequate training in discharge summary writing was reported by 13 (36\%) junior doctors.

Conclusions: Although based on small sample sizes from one location, the level and range of differences in perceived importance of reporting medication changes suggests that many discharge summaries may not currently fulfil GP requirements for managing continuity of care. Results indicate that over a third of junior doctors felt inadequately prepared for writing discharge summaries. There may therefore be both a need and professional support for further training in discharge summary writing, requiring confirmatory research.

Key words: Patient discharge, discharge summary, patient transfer, interdisciplinary communication, medical education

\section{Introduction}

Accurate, comprehensive transfer of information about prescribed medicines across the healthcare interface is essential to ensure consistency between the treatment provided in hospital and in the community, and to ensure patient safety through the avoidance of medication-related inaccuracies. However, deficits in communication are widely reported. ${ }^{1-4}$ In 2009 a national survey of UK primary care General medical Practitioners (GPs) reported that they considered the information received on a discharge summary when a patient is transferred from secondary to primary care to be inadequate. ${ }^{5}$ They had particular concerns about discharge summary accuracy, timeliness and detail regarding medication changes.

UK prescribing guidance, developed following extensive public consultation ${ }^{6}$ states that when patient care is transferred to the GP, secondary care doctors are obliged to provide details of the patient's current and recent medicine use, medicine changes, length of intended treatment, monitoring requirements, and any new allergies or adverse reactions. ${ }^{7}$ Furthermore, in response to GPs raising concerns over receiving discharge information late, after the patient's first post-discharge GP visit, ${ }^{8}$ a reduced timeframe of twenty-four hours after patient discharge for a discharge summary to be received in primary care was imposed from 2008. ${ }^{9}$ GPs have since reported an increased incidence of incomplete or inaccurate discharge information compromising patient safety. ${ }^{10}$

In the UK, preparation of discharge summaries is primarily the responsibility of junior doctors, who are undertaking a two-year foundation training programme between graduating from medical school and undertaking specialist medical training. In 2009, a UK study reported that $90 \%$ of all discharge summary items were written by doctors in their first and second foundation year of train- 
ing. ${ }^{11}$ Although hospital consultants have overall responsibility for the discharge summaries produced, they will rarely check the content. However, a pharmacist carrying out a final check has been demonstrated to improve the quality of discharge summaries. ${ }^{12,13}$

Both junior doctors and medical students have reported receiving inadequate guidance and training on how to write discharge summaries, ${ }^{14,15}$ and recognise that higher priority is often given to more immediately-pressing clinical tasks. ${ }^{16}$ Currently, each UK hospital uses its own unique prescribing system, and so, training of junior doctors in this area is difficult to standardise, with in-house training often being relied upon.

Inadequacies in training for discharge summary preparation may be linked to limited intra-professional understanding between secondary and primary care doctors about discharge summary requirements, which could be a cause of poor quality summaries. The primary aim of this study was therefore to explore and compare the priorities and values of doctors working at either side of the primary and secondary care interface on medicines-related discharge information. Our objectives were to elicit what importance GPs give to the individual content, accuracy and timeliness of discharge summaries, and how their views compare to those of junior doctors.

The secondary objectives were to investigate GP perceptions of and preferences for the timeliness, level of accuracy, pharmacy input and provision of medication changes on discharge summaries. We also investigated what training junior doctors had received on writing discharge summaries.

\section{Method}

\section{Study design}

A questionnaire survey to capture the opinions of both primary and secondary care doctors was undertaken following ethical approval from the University of East Anglia Faculty of Medicine and Health Ethics Committee.

The study site in secondary care was a 600-bed general hospital in the UK which had been employing an electronic discharge system since 2008. This system enables discharge summaries to be generated electronically on the wards and emailed to the patient's GP by the discharging doctor. Discharge summary content is typed manually into the electronic template. The primary care site was a group of 43 GP practices caring for 325,000 patients in the one UK region served largely by the study hospital.

\section{Participants and sample size calculation}

At the time of study completion, 74 junior doctors were employed by the hospital and 173 GPs were located in the study site GP practices. Previous surveys to GPs and junior doctors have reported response rates of around $30 \% .{ }^{17-19} \mathrm{An}$ anticipated response rate of $30 \%$ would give a sample size of 46 GP surveys and 22 junior doctor surveys. For questions eliciting a response between $50 \%$ and $90 \%$, these would provide $95 \%$ confidence intervals of $36 \%$ to $64 \%$ and $81 \%$ to $99 \%$ respectively for GPs, and $29 \%$ to $71 \%$ and $77 \%$ to $100 \%$ respectively for junior doctors. For questions eliciting a response between $50 \%$ and $90 \%$, these would provide $95 \%$ confidence intervals of $36 \%$ to $64 \%$ and $81 \%$ to $99 \%$ respectively for GPs, and $29 \%$ to $71 \%$ and $77 \%$ to $100 \%$ respectively for junior doctors.

\section{Sampling methods}

The survey was posted to all 153 GPs not involved in the piloting stage, and by internal mail to all 74 junior doctors employed by the hospital, together with a covering letter and survey participation card. Each doctor contacted was allocated a unique study reference code, which was printed on a separate survey participation card and sent to doctors alongside the survey. Receipt of a completed participation card indicated a response, thus preventing follow-up, whilst allowing survey answers to remain anonymous. A follow-up copy was sent to non-respondents after two weeks. Failure to respond to the second questionnaire after a further two weeks was treated as non-participation in the study.

\section{Data collection}

The questionnaire was based on four characteristics and four types of content of discharge summaries. Selection of these characteristics and content was informed by the recommended minimum dataset of information to accompany a patient when they transfer between care settings ${ }^{7,20,21}$ and an audit conducted at the study hospital. This audit investigated the accuracy, pharmacy input, timeliness and quality of discharge summaries, and reported particularly poor adherence of summaries to the standards of providing information about medication changes and accurate medication-related information. ${ }^{22}$ Questions conforming to evidence based recommendations were prepared in order to collect both factual and attitudinal data from doctors. ${ }^{23}$

The questionnaire was subsequently reviewed and refined in discussion with a multidisciplinary team comprising supervisory pharmacy practice researchers and a qualitative health researcher at UEA, a health economics researcher with specialist experience in questionnaire design, and senior clinical pharmacists and senior hospital doctors at the secondary care site in order to establish content validity.

The questionnaire comprised three sections totalling 18 items and used a combination of Likert scale, and open responses as recommended for capturing attitudinal data, whilst the yes/no style was primarily used for factual data. ${ }^{24}$, ${ }^{25}$ Different versions were prepared for GPs and secondary care doctors. From GPs, section 1 was designed to capture the following:

- Existing timeliness with which discharge summaries are received, and timeframe GPs considered acceptable for discharge summary receipt 
- Current level of accuracy of discharge summaries and GP practice time spent resolving inaccuracies

- GP perceptions of the importance of a discharge summary being checked for accuracy prior to receipt

- From junior doctors, section 1 was designed to capture the following:-

- Frequency with which they wrote discharge summaries

- Frequency with which junior doctors reported discharge summaries as being checked for accuracy by a pharmacist before releasing to primary care

- Whether junior doctors had received formal training in discharge summary writing. If received, where this had taken place and perceived adequacy

Sections 2 and 3 were identical for both primary and secondary care doctors.

Section 2 cited four characteristics of discharge summaries (timeliness, accuracy, completeness, and, spelling and grammar) and four items of discharge summary content (full list of medicines, medication changes, rationale for medication changes, and medication continuation plans), which doctors were asked to rank in order of importance on a Likert Scale of 1 to 4 , where 1 is most important and 4 is least important. Doctors were asked to choose only one number per characteristic and content. This section contained a further three questions. The first two were open questions inviting comment on:

- Any discharge summary characteristics other than the four listed above perceived as important

- The one change most desired to existing discharge summaries produced at the secondary care study site

The last question in this section was a closed question asking respondents to identify whether details of medicines prescribed at discharge or details of medicine changes during hospitalisation are most important in a discharge summary.

Section 3 asked for information from the respondent about their gender and number of years qualified as hospital or primary care doctor, so as to characterise the respondent sample.

Content and face validity were further established through piloting the questionnaire with 20 randomly selected (using a list of GP reference numbers and a random number generator) GPs based in one UK region. The questionnaires were distributed by post therefore response rate was also estimated. Following piloting, ranking questions were changed, from asking respondents to assign a rank to each discharge summary component (characteristic or content), to asking respondents to draw a line between a list of ranks and components. This made it less likely that doctors would allocate more than one rank to each listed component. Pilot responses were excluded from the main analysis.

\section{Data analysis}

Descriptive statistics were used to report doctors' responses. Fisher's exact test was used to compare the preferences of GPs and junior doctors for pharmacy checking and information provision on medication changes. Ranking choices were compared using Mann-Whitney $U$ test. Simple thematic analysis was used to group and explore free text comments thus providing further depth to the quantitative data. Invalid responses, where the doctor had assigned more than one choice to each rank were excluded from the final analysis.

\section{Results}

\section{Response rates}

Of 232 questionnaires distributed (excluding the pilot), 36 (49\%) junior doctors and 42 (28\%) GPs returned a completed questionnaire.

\section{Training for junior doctors in writing discharge sum-} maries

Twenty-eight (78\%) junior doctors reported receiving formal training for writing discharge summaries, however only $6(19 \%)$ received this as part of their medical degree, and 13 (36\%) felt that the amount they received was inadequate. Mean (Standard Deviation) time spent preparing discharge summaries reported by junior doctors was $27 \%$ (19.2): 33\% (22.5) for junior doctors in foundation year 1 and 19\% (10.2) for junior doctors in foundation year 2.

Six junior doctors raised the need for guidance and training on what information should be included on discharge summaries. All suggested this should be consultant or GP-led as represented by the following quote: "It would be helpful to hear directly from GPs what they need, and what information is useful/not useful to them" (respondent 22). Some specified that they would like this training to include guidance on appropriate content and that good practice examples would be helpful: "I would like to see some examples of what are considered good summaries" (respondent 10) and "some idea of content expectations would help" (respondent 8).

\section{Ranking questions}

All 36 junior doctors answered both the ranking questions for the characteristics and content of discharge summaries, of which 33 (92\%) and 32 (89\%) responses respectively were valid (with only one rank assigned to each item). The same ranking questions were completed by 39 (93\%) and 38 (91\%) GPs respectively, of which 35 (90\%) and 33 (87\%) responses respectively were valid.

\section{Characteristics and content}

Table 1 displays the average rankings assigned to the variables for characteristics and content of discharge summaries. The characteristic deemed most important by the greatest proportion of GPs and junior doctors was 'accuracy', which was assigned a rank of 1 ('most important') 
by $24(73 \%)$ GPs and 28 (88\%) junior doctors; no GPs or junior doctors ranked 'accuracy' as 4 ('least important'). Only 3 (9\%) GPs and no junior doctors ranked 'timeliness' as 'most important'. The content deemed most important by the greatest proportion of GPs and junior doctors was details of 'medication prescribed', which was assigned a rank of 1 by 19 (54\%) GPs and 23 (70\%) junior doctors. 'Medication changes' were ranked as 'most important' by 13 (39\%) GPs compared to 4 (12\%) junior doctors. Statistically significant differences between the ranks assigned to medication changes and continuation plans by GPs and junior doctors were observed (Table 1).

GPs were largely dissatisfied with the quality of information about medication changes provided on discharge summaries, with 25 (61\%) GPs describing details of changes as 'poor' or 'very poor'. No GPs rated details of medication changes as 'excellent'. Details of specific changes to medication, rather than a full list of all the prescribed medication, at discharge was preferred by 20 (49\%) GPs compared to 10 (28\%) junior doctors (Fisher's Exact test, $\mathrm{p}=0.062$ ).

Table 1. Median ranks assigned by doctors to discharge summary characteristics and content listed in the survey

\begin{tabular}{lccc}
\hline \multirow{2}{*}{$\begin{array}{l}\text { Discharge summary } \\
\text { component }\end{array}$} & \multicolumn{2}{c}{ Median (IQ) rank } & $\begin{array}{c}\text { Mann-Whitney } \\
\text { U test }\end{array}$ \\
\cline { 2 - 4 } & GP & $\begin{array}{c}\text { Junior } \\
\text { Doctor }\end{array}$ & p value \\
\hline Characteristics & $\mathrm{n}=33$ & $\mathrm{n}=32$ & \\
$\quad$ Accuracy & $1(1,2)$ & $1(1,1)$ & 0.144 \\
Completeness & $3(2,4)$ & $2(2,3)$ & $0.006^{*}$ \\
Timeliness & $3(2,3)$ & $3(2,3.75)$ & 0.132 \\
$\quad$ Grammar & $3(2,4)$ & $4(3,4)$ & 0.068 \\
Content & $\mathrm{n}=35$ & $\mathrm{n}=33$ & \\
$\quad$ Medication prescribed & $1(1,3)$ & $1(1,2)$ & 0.078 \\
Continuation plans & $3(2,3)$ & $3(2,4)$ & 0.728 \\
Medication changes & $2(1,3)$ & $3(2,3)$ & $0.009^{*}$ \\
Rationale for changes & $3(3,4)$ & $3(3,4)$ & 0.812 \\
\hline
\end{tabular}

*significance at the 0.05 level

\section{Accuracy of discharge summaries}

The median (lower quartile, upper quartile) proportion of summaries which GPs reported to contain inaccuracies requiring practice time to address was $15(10,30)$ percent, with each inaccuracy taking a median (lower quartile, upper quartile) time of $0.5(0.5,1.0)$ hours to resolve. When considering accuracy checking of summaries by a pharmacist, 16 (44\%) junior doctors reported not feeling comfortable with sending unchecked discharge information, whilst 29 (71\%) GPs reported not feeling comfortable using unchecked discharge information to update their records (Fisher's Exact test, $\mathrm{p}=0.023$ ).

A minority of GPs also reported being unaware that pharmacists sometimes do not check the discharge summary: "I had assumed that all the information we receive is checked for accuracy" (GP respondent 22). Others did not know where information about the pharmacy checking status could be found on the discharge summary: "I didn't even notice the box which tells you if this has been done" (GP respondent 21).
All GPs stated values of 24 hours or less for the ideal time in which to receive discharge summaries. However, 24 (59\%) GPs would be willing to wait longer than 24 hours to receive a discharge summary in order to guarantee it had been checked for accuracy.

\section{Discussion}

The present study investigated what importance doctors working at either side of the primary and secondary care interface gave to the individual content, accuracy and timeliness of discharge summaries. Results suggest that while there were some key differences in priorities between the two groups of doctors, the majority of both GP and junior doctor respondents considered accuracy to be the most important characteristic of discharge summaries.

Whilst junior doctors appreciated the importance of discharge information being correct, in practice, a high error rate continues to be observed in discharge summaries. ${ }^{5,26,27}$ Recent research into the causes of prescribing errors by junior doctors at hospitals in the UK has shown that latent conditions (e.g. organisational processes, staffing), error-producing activities (e.g. busy environment, complex patient), active failures (e.g. mistakes) and lack of defences, such as a pharmacy check, can lead to errors being made. ${ }^{11}$ It may be that the environment within which doctors write summaries, the training and information resources available to them, and the possibility for human error introduced by them actually writing the summary, reduce means and time for accurate discharge summaries to be consistently prepared.

\section{Accuracy versus timeliness}

Even though junior doctors reported considering accuracy as more important than timeliness, discharge summaries were often sent without being checked for accuracy by a pharmacist in order to expedite receipt by GPs and to meet the nationally agreed target for sending discharge information within 24 hours. $^{28}$

Whilst timely transfer of information is undoubtedly desirable, the rationale behind the 24 hour government target is unclear, as hospitals will generally supply at least seven days' worth of medicines at discharge, and it is unlikely that a patient will need to visit their GP within 24 hours of being discharged. Currently, there is no UK evidence which supports implementation of the 24 hour target in terms of related patient outcomes. There is UK evidence, however, that improved continuity of care with GPs and structured discharge planning are effective in reducing emergency admissions and re-admissions respectively. ${ }^{29} \mathrm{GPs}$ receiving inaccurate discharge information will disrupt continuity of care and structured discharge planning.

Instead, this time target has placed increased pressure on junior doctors to send out discharge information, often for patients with whom they have had no experience of 
treating, and this may not allow sufficient time for a pharmacist to make a second check of the summary. Whilst junior doctors appear more content to comply, perhaps because their relative lack of experience may limit their understanding of the potential consequences or simply because of it being common practice, most GPs reported that they were not comfortable with using unchecked discharge information. The relative lack of concern expressed by junior doctors for checking the accuracy of summaries may indicate that they assume that GPs will always recognise whether or not the summary has been accuracy checked, or that the GP will provide a second check themselves upon receipt, rather than assuming that all the information provided on the summary is correct. Such assumptions would not be well-founded. GPs reported a preference for summaries to be received within 24 hours, but many would prefer to wait longer to ensure they had been checked for accuracy. A relaxation of the 24 hour target might therefore allow for improvements in the quality of summaries, which they would welcome.

\section{Medication changes}

GPs have consistently been found to value provision of details of medication changes on discharge summaries, which validates the inclusion of this content in the Royal Pharmaceutical Society's recent transfer of care guidance, as part of the minimum dataset recommended to be provided when care is transferred between settings. ${ }^{20}$

GPs more often saw the explicit inclusion of details of medication changes more important than did the junior doctors. Nearly half of GPs preferred receiving only details of medication changes which had been made during the admission to receiving a full list of prescribed medication on discharge summaries, compared to just over a quarter of junior doctors who believed this to be the case. This key difference in priorities could indicate that junior doctors lack awareness of how GPs use information about medication changes for the purpose of updating the patient's medication record after discharge. However, by ranking continuation plans higher than medication changes, junior doctors demonstrated an understanding of the need for care continuity post-discharge.

Both GPs and junior doctors perceived details of the rationale for medication changes as least important. This may be because the rationale for changes made can sometimes be inferred from other discharge information provided, such as diagnosis. However, recent investigation into the documentation of prescribing decisions in a UK hospital found that hospital doctors are often unable to deduce why changes have occurred from the documentation available to them. ${ }^{30}$ Further research to explore how junior doctors gather information about medication, using the resources available to them, when composing discharge summaries is therefore warranted.

\section{Training and guidance}

Reflecting findings from previous literature, ${ }^{14}$ junior doctors described having received little guidance on writing discharge summaries. Junior doctors expressed a desire for more training on the ideal content to include in a discharge summary, indicating a lack of confidence in what is required from them. This is consistent with recent findings from a study of postgraduate trainee medics in Canada, investigating trainees' perceptions of their own and others' roles at discharge, which found a lack of both inter and intra-professional clarity regarding roles and responsibilities. Substantial disagreement between trainees was reported for $38 \%$ of the 13 discharge roles described. ${ }^{31}$

Inter-professional education has been introduced to UK undergraduate healthcare degree programmes and postgraduate courses, to foster "an understanding by every student of the roles of members of different professions in the health and social care team, with a view to ensuring that such teams work more effectively", ${ }^{32}$ and is supported across UK nursing, medicine and pharmacy curricula. ${ }^{32-34}$ The concept of intraprofessional education, however, which facilitates understanding of the roles of other workers within their own profession, is presently under-researched. ${ }^{35}$ In the present study, the GPs' lack of awareness of the process and frequency by which summaries are checked for accuracy, combined with a lack of junior doctor confidence with respect to desirable summary content, suggests that promotion of intra-professional understanding between primary and secondary care doctors might assist in improving the quality of discharge summaries being produced. Further exploratory research in this area is therefore warranted.

\section{Study limitations}

The present study was a small, local service evaluation of a UK general hospital and consequently may not be representative of all hospitals and the GP population they serve within the UK. GPs included were familiar with receiving electronically written and sent discharge summaries and so may have had different views to those using only paperbased summaries.

For the purpose of this study only four different characteristics and content of discharge summaries were selected for examination. These had, however, been identified from existing guidance on the transfer of patient care and previous audit findings as being of relevance to the future research objectives of the team and secondary care organisation. Although doctors were asked to list any other components which they considered to be of importance, they were not asked to rank these additions. Some doctors stated that it was impossible to rank the content listed, and these respondents were excluded from final analysis. In instances where ranking is unsuitable, or where more information than simply a list of ranks is required, application of a Discrete Choice Experiment (DCE), a type of 
stated preference research in which a description of a service is provided according to its distinct specific properties, may be suitable. A DCE would, in the case of this study, enable the relative value of discharge summary components to be examined together with the willingness of doctors to trade between them in order to gain an increase or reduction in particular components.

\section{Conclusion}

Although both GP and junior doctors identify accuracy as the most important characteristic of discharge summaries, junior doctors reported frequently sending information into primary care that had not been accuracy-checked by pharmacy, and worryingly, many seemed comfortable with doing so. The current 24 hour target allows only a minimal timeframe for hospital doctors to produce summaries, and only a narrow window of opportunity in which pharmacists can check summaries to ensure accuracy. Further research to investigate the appropriateness of any relaxation of the 24 hour target is therefore warranted.

Junior doctors reported inadequate training and guidance for the preparation of discharge summaries and their ideal content, which might explain the difference observed between GPs and junior doctors' perceptions of the importance of medication changes to be provided on summaries.

One reason for this could be that junior doctors lack understanding of the GP's role with respect to updating patients' medication list following patients' discharge from hospital. When prioritising work and deciding on the most appropriate actions, it is important to understand the perspective of people affected by those actions. If junior doctors' perceptions of what is important in producing discharge information differ from those of general practitioners, then it is likely that problems will persist. Promotion of intra-professional understanding between the two groups of doctors through the provision of GP-led training might assist in bridging the gap between the two care settings and improving the quality of information produced by hospital doctors at discharge.

\section{Acknowledgements}

We are indebted to Anne Regan, David Green and Lorna Hollister in the pharmacy department at Colchester Hospital University NHS Foundation Trust (CHUFT), and Dr Michela Tinelli at London School of Economics, for their assistance with and comments on the survey design. We thank staff in the postgraduate medical centre at CHUFT who provided access and helped to organise survey distribution to junior doctors. This project was conducted as part of a $\mathrm{PhD}$ jointly funded by the University of East Anglia and Colchester Hospital University NHS Foundation Trust.

\section{Conflict of Interest}

The authors declare that they have no conflict of interest.

\section{References}

1. Penney T. Delayed communication between hospitals and general practitioners: where does the problem lie? British Medical Journal. 1988;297(6640):28.

2. Kripalani S, LeFevre F, Phillips CO, Williams MV, Basaviah P, Baker DW. Deficits in communication and information transfer between hospital-based and primary care physicians: implications for patient safety and continuity of care. JAMA. 2007;297(8):831-41.

3. Moore C, Wisnivesky J, Williams S, McGinn T. Medical errors related to discontinuity of care from an inpatient to an outpatient setting. Journal of General Internal Medicine. 2003;18(8):646-51.

4. Van Walraven C, Weinberg A. Quality assessment of a discharge summary system. Canadian Medical Association Journal. 1995;152(9):1437.

5. Care Quality Commission. Managing patients' medicines after discharge from hospital. London: Care Quality Commis-sion; 2009.

6. GMC issues new prescribing guidance [Press Release]. General Medical Council; 2013 [cited 27 February 2014]; Available from: http://www.gmcuk.org/news/14346.asp.

7. General Medical Council. Good practice in prescribing and managing medicines and devices. London: General Medical Council; 2013.

8. NHS Alliance. A very present danger. A national survey into information provided by hospitals to GPs when patients are discharged. London: NHS Alliance; 2007.

9. Department of Health. Standard terms and conditions for acute hospital service 2011/12. London: Department of Health; 2010.

10. NHS Alliance. Warning: danger ahead! Report of the $4^{\text {th }}$ NHS Alliance national survey into patient discharge information. London: NHS Alliance; 2010.

11. Dornan T, Ashcroft D, Heathfield H, Lewis P, Miles J, Taylor D, et al. An in depth investigation into causes of prescribing errors by foundation trainees in relation to their medical education. EQUIP study. London: General Medical Council; 2009.

12. Abdel-Qader DH, Harper L, Cantrill JA, Tully MP. Pharmacists' interventions in prescribing errors at hospital discharge: an observational study in the context of an electronic prescribing system in a UK teaching hospital. Drug Safety 2010;33(11):1027-44.

13. Campbell F, Karnon J, Czoski-Murray C, Jones R. A systematic review of the effectiveness and cost-effectiveness of interventions aimed at preventing medication error (medicines reconciliation) at hospital admission. The University of Sheffield, School of Health and Related Research (ScHARR); 2007 [cited 19 June 2014]; Available from: http://www.nice.org.uk/nicemedia/pdf/patientsafetymedssystematicreview. pdf.

14. Heaton A, Webb DJ, Maxwell S. Undergraduate preparation for prescribing: the views of 2413 UK medical students and recent graduates. British Journal of Clinical Pharmacology. 2008;66(1):128-34.

15. Maxwell S, Walley T. Teaching safe and effective prescribing in UK medical schools: a core curriculum for tomorrow's doctors. British Journal of Clinical Pharmacology. 2003;55(6):496-503.

16. Frain JP, Frain AE, Carr PH. Experience of medical senior house officers in preparing discharge summaries. British Medical Journal. 1996;312:350-1.

17. Bonevski MP, Horton G, Foster M, Girgis A. Response rates in GP surveys - trialling two recruitment strategies. Aust Fam Physician. 2011;40(6):427-30.

18. Hann DM, Sibbald PB. General Practitioners' attitudes towards patients' health and work. Sheffield: Department of Work and Pensions; 2011.

19. Gilbert A, Hockey P, Vaithianathan R, Curzen N, Lees P. Perceptions of junior doctors in the NHS about their training: results of a regional questionnaire. BMJ Quality \& Safety. 2012;21(3):234-8.

20. Royal Pharmaceutical Society. Keeping patients safe when they transfer between care providers - getting the medicines right. London: Royal Pharmaceutical Society; 2011.

21. National Prescribing Centre. Medicines reconciliation: a guide to implementation. Liverpool: National Prescribing Centre; 2008.

22. Yemm R, Wright D, Hollister L, Green D, Wood J. Investigating medication errors on Electronic Discharge summaries: their nature, severity and predictors. International Journal of Pharmacy Practice. 2012;20:54-5. 
23. Bulmer M, De Vaus DA, Fielding N. Questionnaires. London: Sage Publications; 2004

24. Sudman S, Bradburn, N.M. Asking Questions: A Practical Guide to Questionnaire Design. London: Jossey-Bass; 1982.

25. Oppenheim AN. Questionnaire design, interviewing and attitude measurement. New York: Pinter; 1992.

26. Grimes T, Delaney T, Duggan C, Kelly JG, Graham IM. Survey of medication documentation at hospital discharge: implications for patient safety and continuity of care. Irish Journal of Medical Science. 2008;177:937.

27. McMillan TE, Allan W, Black PN. Accuracy of information on medicines in hospital discharge summaries. Internal Medicine Journal. 2006;36(4):221-5.

28. Department of Health. Standard NHS contract for acute services 2008/09. London: Department of Health; 2007.

29. Purdy S. Avoiding hospital admissions: what does the research evidence say? London: The King's Fund; 2010.

30. Tully M, Cantrill J. Insights into creation and use of prescribing documentation in the hospital medical record. Journal of Evaluation in Clinical Practice. 2004;11(5):430-7.

31. Card SE, Ward HA, Chipperfield D, Sheppard M. Postgraduate internal medicine residents' roles at patient discharge - do their perceived roles and perceptions by other healthcare providers correlate? Journal of Interprofessional care. 2014;28(1):76-8.

32. Levison D. CHMS Position Paper: Interprofessional Education. London: Council of Heads of Medical Schools and Deans of UK Faculties of Medicine; 2003.

33. Clifton M, Dale C, Bradshaw C. The impact and effectiveness of Interprofessional education in primary care: an RCN literature review. London: Royal College of Nursing; 2006.

34. General Pharmaceutical Council. The accreditation of pharmacy courses leading to registration and annotation in Great Britain. London: General Pharmaceutical Council; 2013.

35. Martin GP, Currie G, Finn R. Reconfiguring or reproducing intraprofessional boundaries? Specialist expertise, generalist knowledge and the 'modernization' of the medical workforce. Social Science \& Medicine. 2009;68(7):1191-8. 